\title{
Automatic Detection of Paroxysms in EEG Signals using Morphological Descriptors and Artificial Neural Networks
}

\author{
Christine F. Boos, Fernando M. de Azevedo \\ Geovani R. Scolaro and Maria do Carmo V. Pereira \\ University Federal of Santa Catarina
}

Brazil

\section{Introduction}

The first recordings of brain activity in the form of electrical signals, through the use of a galvanometer and insertion of two electrodes on the scalp of an individual, were conducted in 1875 by British scientist Richard Caton. Since then, the electroencephalogram (EEG) has been used to denote the neural electrical activity of the brain (Sanei \& Chambers, 2007).

The EEG signal acquisition can be performed by introducing electrodes inside the brain tissue (depth EEG), the placement of electrodes directly into the exposed surface of the cerebral cortex (electrocorticogram - ECoG) or the positioning of electrodes in a noninvasive, on the surface of the scalp (scalp EEG - sEEG).

The human scalp EEG, in relation to its physical characteristics, occupies a frequency band of $0 \mathrm{~Hz}$ to $100 \mathrm{~Hz}$ and an amplitude range of $2 \mu \mathrm{V}$ to $200 \mu \mathrm{V}$, however, in general, the signal is concentrated between $0,5 \mathrm{~Hz}$ and $60 \mathrm{~Hz}$ with an average amplitude of $50 \mu \mathrm{V}$ (Coimbra, 1994).

These frequency and amplitude value of the EEG signal are influenced by a series of characteristics, such as: location in the cerebral cortex from where recording was acquired, age of the subject, physical (sleep, wake, coma, etc.) and behavioral (depression, excitement, euphoria, stress, etc.) state of the subject. Furthermore, the signal can also be distorted by artifacts interference from many sources, for example, extra cerebral electrical potentials from the patient, the electrodes, the signal acquisition system and external electromagnetic interferences. This means that the recordings may have variability among patients under identical circumstances and in the same patient over time. The presence of this variability contributes to the great difficulty presented in attempts to mathematically model the electrographic patterns commonly present in the EEG signal. Nevertheless these patterns have a relative regularity in frequency, morphology and amplitude - whichever they are i.e. the frequency spectrum of the EEG is usually divided into frequency bands that may be related to different physical states and behavior (Sanei \& Chambers, 2007):

- alpha band - 8 to $13 \mathrm{~Hz}$ - common rhythm in normal patients and more easily observable while the subject is awake, relaxed and with its eyes closed;

- beta band - 13 to $22 \mathrm{~Hz}$ - common rhythm in normal adult patients during wakefulness. Dominant in the pre-central region of the brain but also occurs in other brain regions. Subdivided into: beta I, 13 to $17 \mathrm{~Hz}$ - present during intense 
activation of central nervous system (CNS) - and beta II, 18 to $22 \mathrm{~Hz}$ - decreases during intense activation of the CNS;

- theta band - 4 to $8 \mathrm{~Hz}$ - rhythm frequent in children, in central and temporal region of the brain. Typical of early stages of sleep. Some transient components of this rhythm have been found in normal adult patients;

- delta band - 0,5 to $4 \mathrm{~Hz}$ - common rhythm in children, especially infants, in a state of deep sleep. The presence of this wave in adults under a state of alert may indicate abnormalities.

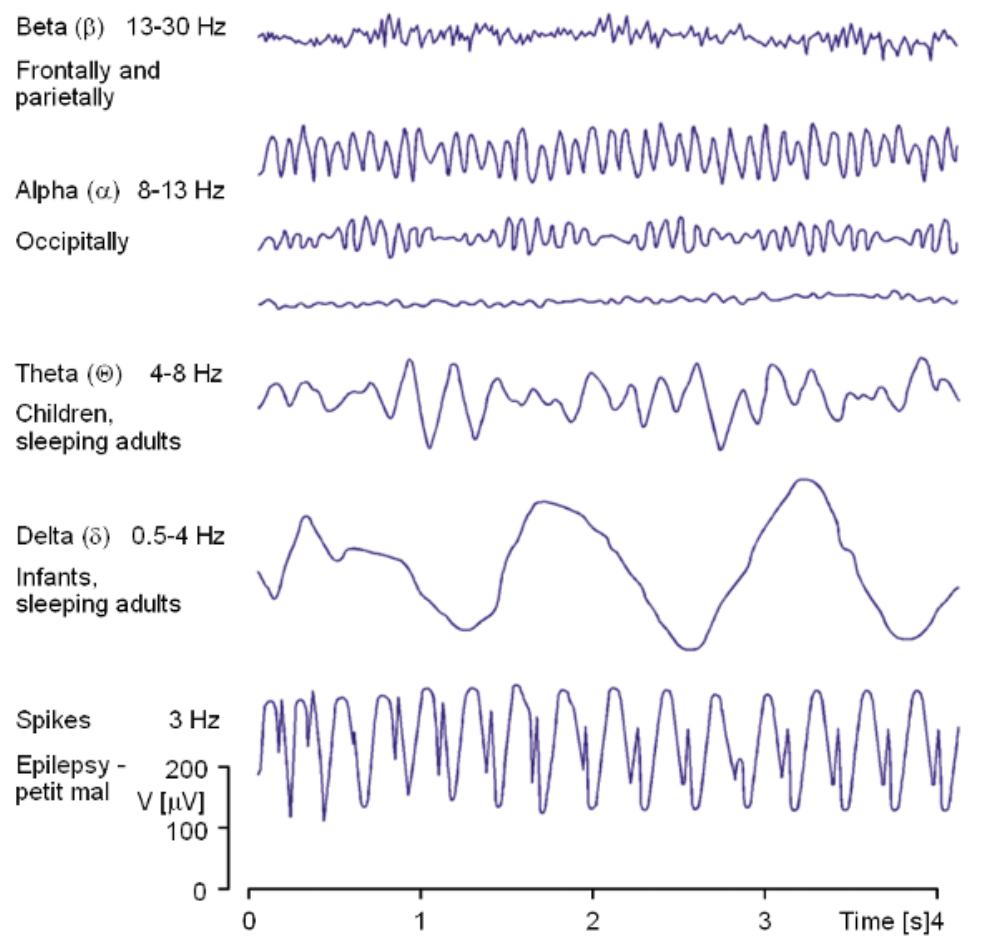

(Sanei \& Chambers, 2007)

Fig. 1. Examples of common electrographic patterns present in the EEG signal

The EEG recording has applicability on, among others: monitoring alertness, coma and brain death; locating damaged areas after head injury, stroke and tumor; testing afferent pathways; monitoring anesthesia depth; researching physiology and sleep disorders; researching epilepsy and localizing the seizure focus (Sanei \& Chambers, 2007).

Epilepsy is a disorder of the brain characterized by an enduring predisposition to generate epileptic seizures and by the neurobiological, cognitive, psychological and social consequences of this condition. The definition of epilepsy requires the occurrence of at least one epileptic seizure, which is a transient occurrence of signs and/or symptoms due to abnormal excessive or synchronous neuronal activity in the brain (Fisher et al., 2005).

Neurologists can make a diagnosis of epilepsy simply through anamneses however EEG signal analysis is a commonly used and important tool to: clinical diagnosis, support in 
defining the type of epilepsy syndrome, provide information for planning drug therapy and also help in deciding the feasibility of surgical intervention.

The occurrence of certain electrographic events, called epileptiform events, in EEG signal is a strong indicator of this pathology presence. An expert in reading these records (EEGer) spends a considerable amount of time reviewing each record, especially when they are acquired for long term monitoring (more than 24 hours) and with many channels - between 24 and 128 leads (Pillay \& Sperling, 2006).

In attempt to facilitate the analysis of EEG recordings by neurologists (or other experts) many studies have proposed automated systems for this analysis. Unfortunately few of them are actually being used worldwide mainly because the available systems for automatic detection of epileptiform events have a relative high number of false identifications (false positives), resulting in little or no effective time save for the process (Wilson \& Emerson, 2002). This means that the low specificity of the systems still discourages EEGers to delegate the task of thorough analysis of the thousands of EEG screens (each with up to 128 continuous signals) in the search for events whose maximum length is $200 \mathrm{~ms}$ and the amplitude values are in the range of microvolts $(\mu \mathrm{V})$.

\section{Automatic detection of paroxysms}

The Biomedical Engineering Institute (IEB-UFSC) of the Federal University of Santa Catarina (UFSC) has as one of their areas of expertise and interest the acquisition, analysis and processing of bioelectrical signals, with emphasis in electroencephalogram (EEG), electrocardiogram (ECG), electromyogram (EMG) and electrooculogram (EOG) signals. Currently within this area there are two research lines, both using neural networks, for automatic detection of epileptiform events: one applies methodologies based on Wavelet Transform and the other one uses a parameterization ${ }^{1}$ of the EEG signal.

\subsection{Approaches based on wavelet transform}

The Wavelet Transform is a tool that allows you to make a more comprehensive analysis of the signal due to the fact that it is possible to obtain information in both time and frequency domains simultaneously. This Transform is a powerful tool for the analysis of nonstationary signals (Wilson et al., 2004) making it ideal for EEG signal analysis. Its basic principle of operation is extract approximation and detail coefficients of the signal at each decomposition carried out, i.e., get high and low frequencies features of the signal for each level of decomposition. Wavelet Transform can be used for the parameterization, filtering and/or feature extraction of the EEG signals and also be very involved in the construction of hybrid intelligent systems for the automatic detection of epileptiform events, providing relatively good results when applied as a preprocessor for Artificial Neural Networks (Kalayci \& Özdamar, 1995; Hoffmann et al., 1996; Oweiss \& Anderson, 2001; Quiroga et al . 2001; Adeli et al., 2003; Khan \& Gotman, 2003; Argoud et al., 2006, Pang et al., 2003, Liu et al., 2006; Argoud et al., 2006; Mohamed et al., 2006; Subasi, 2007; Indiradevi et al., 2008; Ocak, 2008; Abibullaev et al., 2009th, 2009b; Ocak, 2009; Scolaro \& Azevedo, 2010).

\footnotetext{
${ }^{1}$ In this study, the term parameterization refers to the representation of an EEG signal by means of parameters related to morphological characteristics of this signal and these parameters will be called morphological descriptors.
} 


\subsection{Approaches based on parameterization of the signal}

A comparative study of various algorithms used in automatic detection methods, conducted by Wilson and Emerson in 2002, showed that the methods use some form of parameterization of the EEG signal usually get good results.

The first studies involving the parameterization as a tool for the detection of epileptiform events in EEG recording were published by Gotman and Gloor (Gotman, 1976; Gotman \& Gloor, 1982) followed by the research of Webber (1994), Walckzak \& Nowack (2001), Litt (2001) and Tzallas et al. (2006) among others that have obtained promising results.

However with the advances in mathematical methods and the increasing capacity of computer processing the investigations were directed to other approaches (Halford, 2009), for example, the Wavelet Transform, entropy, statistical methods and/or a combination of these and other methods (Kaneko et al. 1999; Diambra, 1999, Liu et al., 2002; Saab \& Gotman, 2005; Tzallas et al., 2006; Übeyli, 2009; Kumar, 2010). Nevertheless we did not abandon the parameterization approach (Guedes et al., 2002, Pereira 2003, Pereira et al., 2003; Sovierzoski, 2009, Boos et al., 2010a, 2010b).

According to the literature, so far one of the most used and successful methods applied in systems for automatic detection of paroxysms is Gotman's (Hoef et al., 2010). This method performs spike modeling through parameters, that in this work will be called morphological descriptors'2, before detection. Gotman's method deals with the EEG signal by dividing it into segments and sequences, both ascending and descending, which are categorized by duration, absolute amplitude and length variation coefficient (which gives information on the cadency of the EEG). In this system, the detection of a paroxysm occurs when the descriptors' values for each epoch exceeds a pre-determined threshold.

Although the literature allows access to various studies that use morphological descriptors to characterize the EEG signal, it is necessary a detailed analysis of the applicability, relevance and effectiveness of each descriptor that will be used.

Therefore our objective is to discuss a methodology for the preparation and evaluation of a set of descriptors for modeling paroxysms through the use of descriptors that are already available in the literature as well as others proposed by us in attempt to improve the differentiation between epileptiform events and other electrographic manifestations that occur in the signal.

\section{Methodology}

This section will present the recordings and methodologies used for both the development of the descriptors' ensemble and the experiments used as an evaluation tool for the proposed set.

\subsection{EEG recordings}

All of the EEG signals used in this study belong to a database with nine records acquired from seven adult patients with confirmed diagnosis of epilepsy. They have a sampling frequency of $100 \mathrm{~Hz}$ and were acquired through 24 (1 record) and 32 channels (8 records).

A bipolar montage (Fig. 2.) type zygomatic-temporal (Zygo-Db-Temp) was used, with 25 electrodes in positions Zy1, Zy2, Fp1, Fp2, F3, F4, F7, F8, F9, F10, CZ, C3, C4, T3, T4, T5, T6,

\footnotetext{
2 The use of the term morphological descriptor is because we believe that this term is more appropriate within the context of parameters referring to morphological characteristics of a signal.
} 
T9, T10, P3, P4, P9, P10, O1, O2 of the 10/20 system and two electrodes positioned for acquisition of electrooculogram (EOG).

For the acquisition process the signals went through analog filtering to isolate the range of 0,5 to $40 \mathrm{~Hz}$. We also observed the need to perform additional filtering to remove the baseline wandering effect (DC frequency - $0 \mathrm{~Hz}$ ) and eliminate noise caused by power line interference $(60 \mathrm{~Hz})$, and it was necessary to perform interpolation of the signal to a sampling frequency of $200 \mathrm{~Hz}$.
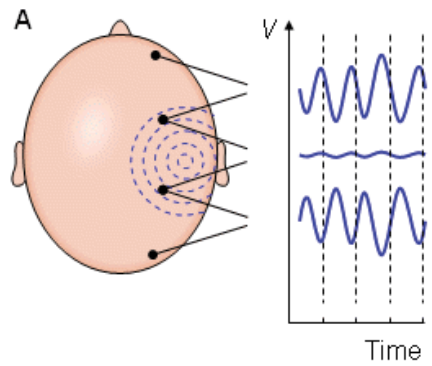

B
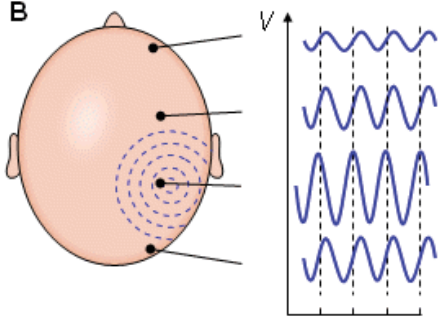

Time

(Malmivuo \& Plonsey, 1995)

Fig. 2. EEG signal differences presented when a bipolar (A) and unipolar or referential (B) montage is used. In the bipolar montage the signal is a result of potential difference between pairs of electrodes while for the unipolar montage the signal is obtained by the difference in potential between an electrode and a reference point (equal for the whole montage)

\subsection{Morphological descriptors}

The literature on the automatic detection of epileptiform events contains a considerable amount of morphological descriptors used in different methodologies and/or developed systems. For our experiments we selected the descriptors most reported in literature: the maximum amplitude of the event, event duration, the length variation coefficient, crest factor and entropy.

The maximum amplitude and duration of the event are self-explanatory. The length variation coefficient - used to measure the regularity of the signal - is the ratio of standard deviation and the mean value of the signal. The crest factor is the difference between the maximum and minimum amplitudes, divided by the standard deviation (Webber et al., 1994). The entropy, reported in several studies - e.g. Quiroga (1998), Esteller (2000), Srinivasan et al. (2007) and Naghsh-Nilchi \& Aghashahi (2010) - provides a value for the complexity of the signal under analysis.

These descriptors are widely used, however they may not guarantee the complete differentiation between the events presented by the recordings and also because of this the existing systems for automatic detection have only a moderate performance. Thus, through a detailed analysis of the EEG signals that are being used, new descriptors based on the physical and/or morphological signal can be developed in attempt to improve the performance of the automatic detection process.

The main focus for the development of new descriptors was to find characteristics in the EEG signals that further highlighted the epileptiform events from other types of events. The latter are called non-epileptiform events (Fig. 3.) and for our database they are represented by: 
a. normal background EEG activity;

b. alpha waves;

c. blinks;

d. artifacts originated from EMG (muscle activity), external electromagnetic interference, among others.
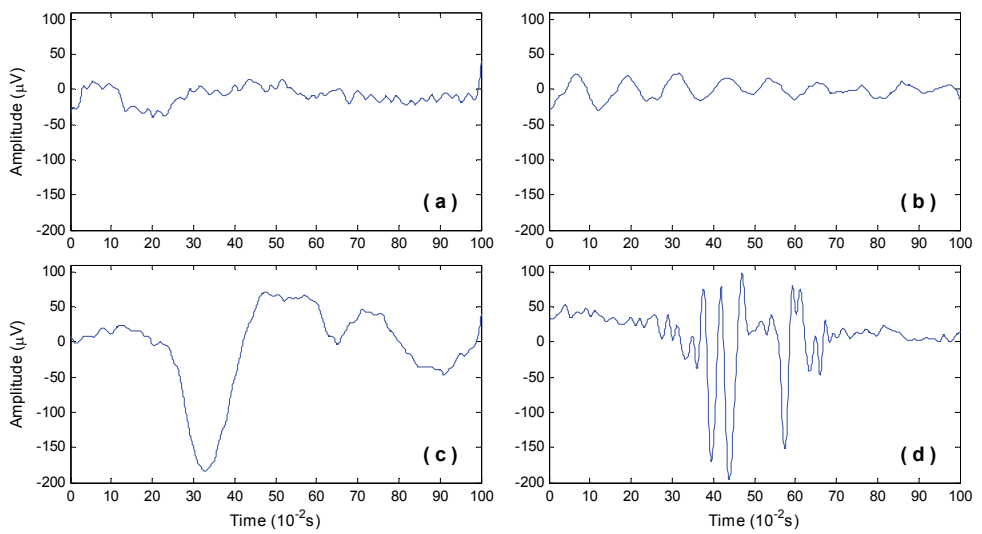

Fig. 3. Morphology of the main non-epileptiform events found in our EEG signals database
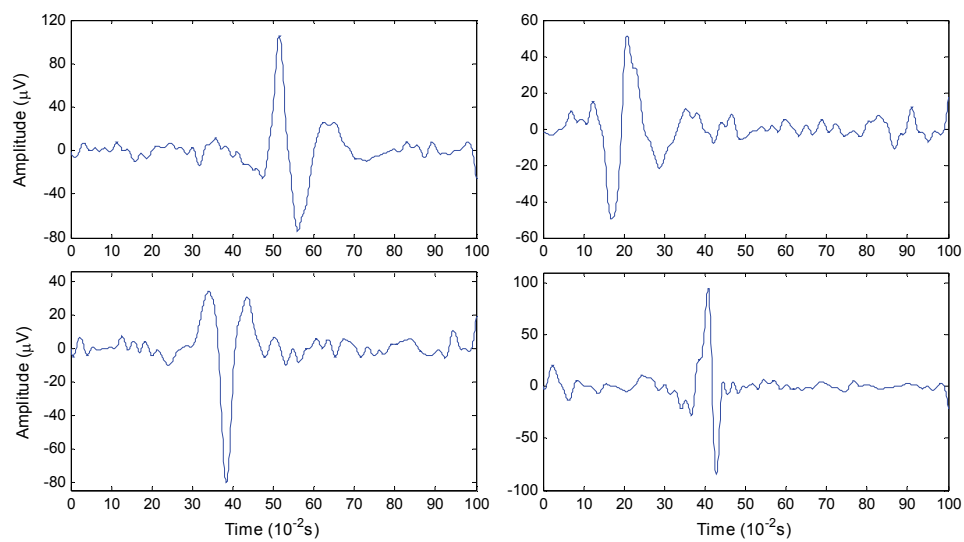

Fig. 4. Morphology presented by the epileptiform events in the recordings under analysis

Looking at the obtained records we realized that due to the use of a bipolar montage (Fig.2) the epileptiform events can appear in four different ways (Fig. 4.). In other words, because of the type of montage the spikes and sharp waves may appear with both electronegative and electropositives amplitude peaks, however to be considered a paroxysm they still have to be followed by a slow wave.

The basic morphological characteristics of an epileptic event are related to their amplitude and duration. The spikes have duration of 20 to $70 \mathrm{~ms}$, while a sharp wave has duration of 70 to $200 \mathrm{~ms}$. Since both events can be a paroxysm and making a distinction between them makes little sense from a clinical point of view, we can consider that the duration 
epileptiform events varies from 20 to $200 \mathrm{~ms}$. The amplitudes values of both spikes and sharp waves are also varied but when considering them epileptiform events the amplitude (module value) usually lies between $20 \mu \mathrm{V}$ and $200 \mu \mathrm{V}$ (Niedermeyer, 2005). Examples of morphological descriptors related to the amplitude and duration of a typical epileptiform event are (Fig. 5.):

- maximum amplitude (Amax);

- $\quad$ minimum amplitude (Bmin);

- difference between the points of occurrence of extreme amplitude (Tdif);

- difference between the maximum and minimum amplitudes (DifAB).

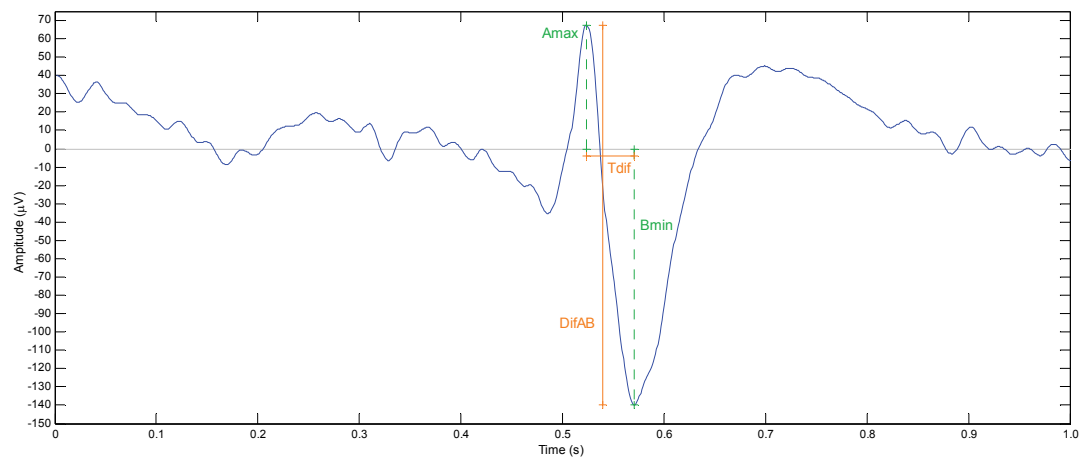

Fig. 5. Morphological descriptors related to the amplitude and duration of paroxysms

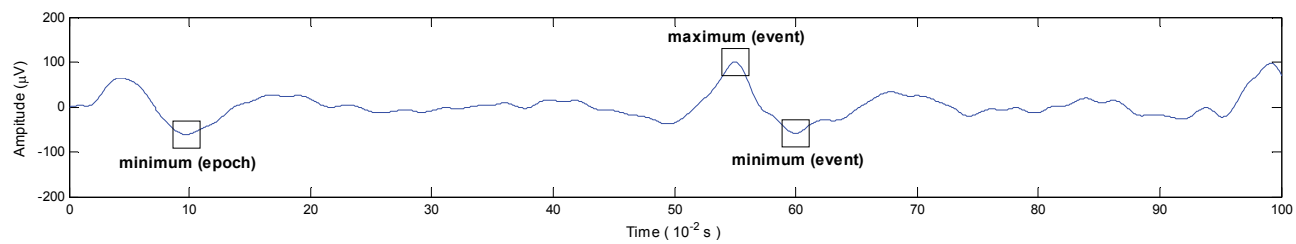

Fig. 6. EEG signal presenting maximum amplitude corresponding to an epileptiform event and minimum amplitude corresponding to another (different) event

Also regarding the amplitudes within the epoch under review (in this case 1 second of the signal) the points of maximum and minimum amplitude may not belong to the same event (Fig. 6.). Analyzing this fact, we could see that to be a paroxysm (event we want to correctly identify) the event should have a time difference between maximum and minimum amplitudes in the range of 35 to $100 \mathrm{~ms}$ (half duration the slowest event). For this, as illustrated in Fig 7, we determined a 300ms segment centered at the event appearing in the epoch under review and within this segment we calculated the following descriptors: maximum amplitude (Amax_pts); minimum amplitude (Bmin_pts); distance between extreme amplitudes (DifAB_pts) and time difference (Tdif_pts) between the maximum and minimum amplitudes.

Another feature that can be observed is that an epileptiform event, particularly the spike, has more acute peaks when compared to the obtuse peaks of alpha waves or blinks (Fif. $3 \mathrm{~b}$ and Fig. 3c). This fact allows another opportunity to discriminate between events since the 


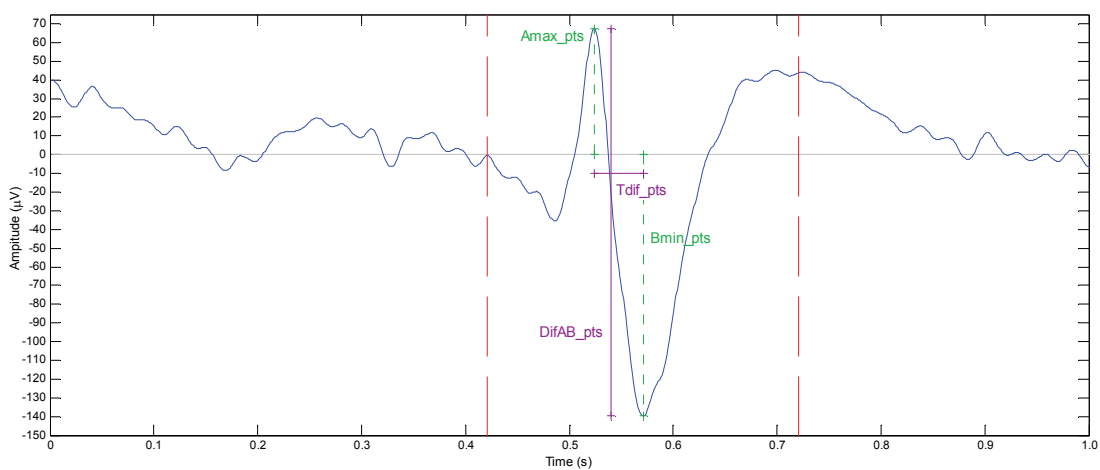

Fig. 7. Maximum amplitude (Amax_pts), minimum amplitude (Bmin_pts), distance between extreme amplitudes (Tdif_pts) and time difference between amplitudes (DifAB_pts), all within the $300 \mathrm{~ms}$ segment centered on the event under analysis

process of automatic detection can confused them, which is a detrimental factor to the system performance. Based on these observations we analyzed the vertex angle of the peaks through the extreme amplitudes and zero crossing points adjacent to the beginning and the end of the event.

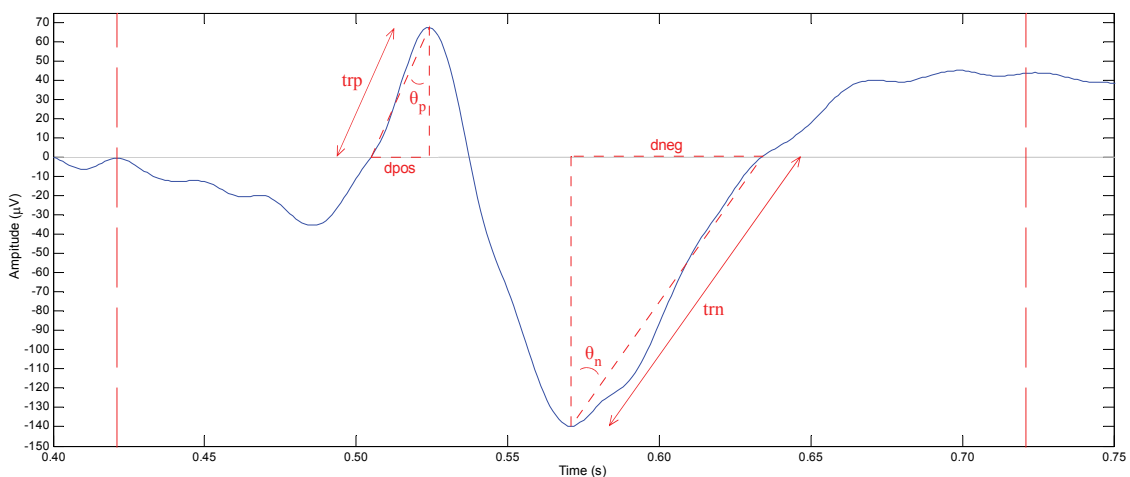

Fig. 8. Vertex angle of positive and negative epileptiform event, calculated from the maximum and minimum amplitude, respectively

The calculated angles (Fig. 8.), taking an epileptiform event as example, refer to the angle influenced by the peak's initial inclination and the angle that suffers influence of beginning slope of the slow wave. Based on the calculation of these angles $(\theta p$ and $\theta n)$ we determined other descriptors:

- base of the peaks directly adjacent to the beginning and the end of the event (dpos and dneg, depending in order of appearance of the peaks);

- $\quad$ angle of the analyzed event apex $(\theta)$;

- tangents of the angles of peak apex (tgp and tgn);

- tilt of the slopes directly adjacent to the beginning and the end of the event (trp and trn);

- $\quad$ event basis (dbase). 
The morphology of a paroxysm can also often be confused with the morphology of artifacts (from various sources) present in the EEG signal. However, as can be seen in Fig. 9. the typical waveforms of these noises usually have a relative high frequency. This means that the high amplitudes appear with minimum time differences between them, which are the opposite of paroxysms that usually have more widely spaced peaks because they are always followed by a slow wave.

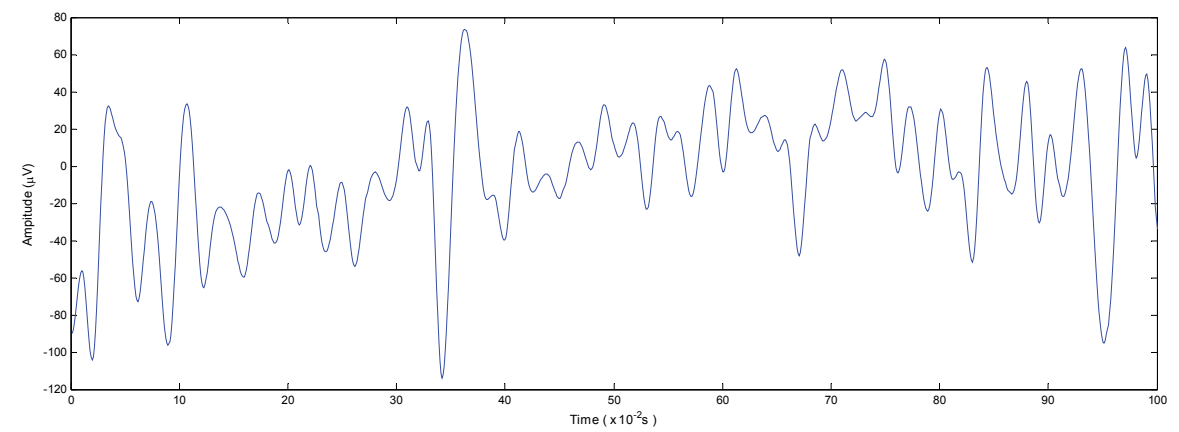

Fig. 9. Example of typical morphology of an artifact (noise) present in the EEG signal

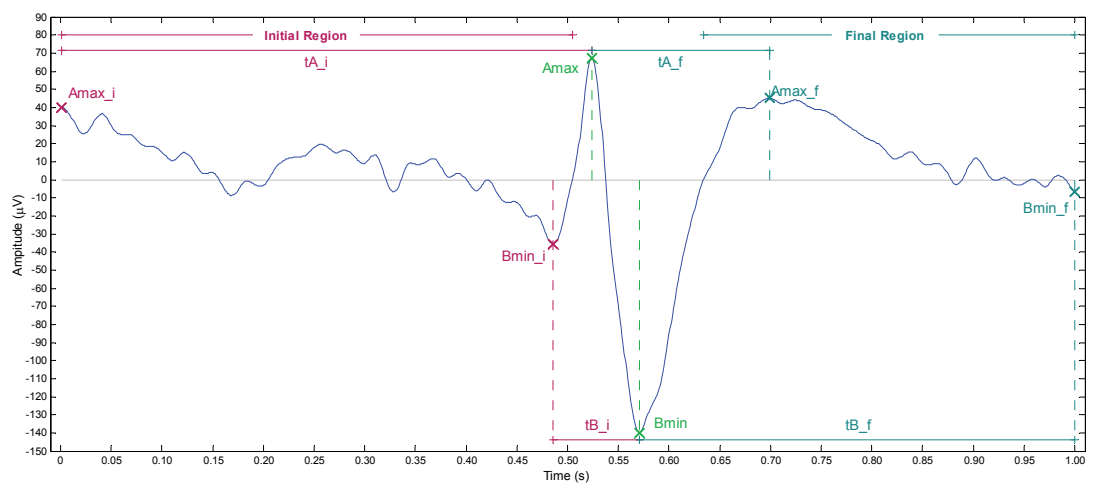

Fig. 10. Descriptors for the differentiation between epileptiform events and artifacts, considering distances (time) between the points of maximum and minimum amplitude

The descriptors proposed to make the distinction between noise and epileptiform events can be based on relations of time and amplitude differences in the epoch when dividing it in two regions (initial and final) adjacent to the event. Experiments were performed and from them we projected the following descriptors:

- amplitude and time difference between maximum amplitudes of the event (Amax), initial (Amax_i) and final regions (Amax_f): DifA_i, tA_i, DifA_f and tA_f;

- $\quad$ amplitude and time difference between minimum amplitudes of the event (Bmin), initial (Bmin_i) and final regions (Bmin_f): DifB_i, tB_i, DifB_f, tB_f.

Further analysis of the morphology and other characteristics of events that occur in the EEG recordings can be performed. In this research it is proposed only the addition of descriptors based on the classical statistical indices of average, standard deviation and variance. These 
descriptors, were calculated for both the epoch under analysis (one second) and the $300 \mathrm{~ms}$ segment. Thus, considering the descriptors selected from the literature and those we developed after a review of the recordings, we obtained a final set of 45 morphological descriptors (Table 1).

\begin{tabular}{|c|c|}
\hline Origin if the descriptors & Descriptors identifications \\
\hline Amplitude & Amax, Bmin, DifAB, Amax_pts, Bmin_pts, DifAB_pts \\
\hline Duration & Tdif, Tdif_pts, T \\
\hline Vertex angle of the peaks & $\theta, \theta p, \theta n$, dbase, dpos, dneg, trp, trn, tgp, tgn \\
\hline Initial region of the epoch & Amax_i, Bmin_i, DifA_i, tA_i, DifB_i, tB_i \\
\hline Final region of the epoch & Amax_f, Bmin_f, DifA_f, tA_f, DifB_f, tB_f \\
\hline Statistical indexes ${ }^{1}$ & $\begin{array}{c}\text { desvio, media, var, coef, CF, desvioC, mediaC, varC, coefC, } \\
\text { CFC }\end{array}$ \\
\hline Entropy ${ }^{1,2}$ & entrop_log, entrop_norm, entrop_logC, entrop_normC \\
\hline
\end{tabular}

${ }^{1}$ The letter ' $C^{\prime}$ at the end of the identification means that the descriptor was calculated for the segment of $300 \mathrm{~ms}$.

${ }^{2}$ We calculated two types of entropy: normalized (norm) and logarithm of "energy".

Table 1. Summary of the elements that compose the final set of 45 morphological descriptors selected and developed for this research

\subsection{Morphological descriptors evaluation}

In the previous item (3.2) 45 morphological descriptors were presented. Some of them were chosen among those universally used and others were defined in our previous work.

After the creation of the descriptors' set it is necessary to analyze this ensemble in order to verify the significance of each element of the group in the differentiation of events. For this research we chose to use correlation analysis and application of Hotelling's T ${ }^{2}$ test (Härdle \& Simar, 2007) for individual assessment and Artificial Neural Networks (Eberhart \& Dobbins, 1990; Zurada, 1992; Haykin, 1994 ) to verify the complete set performance.

The correlation analysis was made evaluating the correlation matrices of descriptors for pairs of events. We examined the correlation between morphological descriptors calculated from epochs containing paroxysm and epochs with non-epileptiform (blinks, artifacts, alpha waves and background EEG activity). The criterion for possible exclusion of any element (descriptor) of the designed set was the existence of high correlation values (above 50\%) for all pairs of events considered.

The Hotelling's $\mathrm{T}^{2}$ test consisted in calculating the difference between the values of each descriptor in epochs with epileptiform transients and epochs with non-epileptiform events. The assessment of this test was made comparing the results of these differences with a predetermined $\mathrm{T}^{2}$ critical value (a threshold). Based on this test a descriptor is considered relevant when its $\mathrm{T}^{2}$ result is greater than the pre-determined critical value.

Some descriptors such as the tangents of the positive $(\theta p)$ and negative $(\theta n)$ angles, length variation coefficient (coef) and crest factor (CF) had $\mathrm{T}^{2}$ test result relatively close to the critical value and thus these elements could have been removed from the set. However as the correlation value achieved by these same descriptors was not high and their exclusion did not affect significantly the sensitivity and specificity of the neural networks implemented in this study. We chose to not exclude them from the final set. 
For the verification that the descriptors can indeed provide sufficient information so a classifier can make the discrimination between events the set was arranged at the input of several Artificial Neural Networks.

The networks used are all Feedforward Multilayer Perceptron with Backpropagation algorithm and supervised learning. The basic architecture of each network was of an input layer with 45 neurons and output layer with only one neuron. The number of neurons in the hidden layer and the application of input stimuli normalization ${ }^{3}$ were varied in each of the networks so we could find the best configuration and analyze the effect of this normalization. Some other features of neural networks implemented are:

- $\quad$ activation function of output and hidden layers: hyperbolic tangent;

- number of neurons in the hidden layer $(\mathrm{N}): 7$ to 11 neurons;

- batch update of the synaptic weights (after every training epoch);

- learning rate and momentum were respectively: 0,01 and 0,9.

Finally, the training and test of networks were made with two different compositions of files (Table. 2.): a set of files classified only by the presence or absence of paroxysms and another set where the files were classified by type of event (sharp waves, spikes, blinks, normal background EEG activity, alpha waves and artifacts).

\begin{tabular}{|c|c|c|c|c|}
\hline Composition & Process & \multicolumn{2}{|c|}{ Signal classification used } & $\begin{array}{l}\text { No. of } \\
\text { files }\end{array}$ \\
\hline \multirow{4}{*}{ Composition I } & \multirow{2}{*}{ Training } & \multicolumn{2}{|l|}{ Epileptiform event } & 47 \\
\hline & & \multicolumn{2}{|l|}{ Non-epileptiform event } & 73 \\
\hline & \multirow{2}{*}{ Test } & \multicolumn{2}{|l|}{ Epileptiform event } & 30 \\
\hline & & \multicolumn{2}{|l|}{ Non-epileptiform event } & 23 \\
\hline \multirow{6}{*}{ Composition II } & \multirow{6}{*}{$\begin{array}{l}\text { Training } \\
\text { and test }\end{array}$} & \multirow{2}{*}{ Epileptiform event } & Sharp wave & 10 \\
\hline & & & Spike & 10 \\
\hline & & \multirow{4}{*}{ Non-epileptiform event } & $\begin{array}{l}\text { EEG background } \\
\text { activity }\end{array}$ & 5 \\
\hline & & & Alpha waves & 10 \\
\hline & & & \begin{tabular}{|l|} 
Artifacts \\
\end{tabular} & 5 \\
\hline & & & Blinks & 5 \\
\hline
\end{tabular}

Table 2. Composition of files according to different classifications of EEG signals events, used for training and tests of the neural networks created

\section{Results}

Several networks with the same basic architecture and features showed in the previous section were trained and tested using both types of file composition (Table 2.). The normalization of input stimuli was tested in all implemented networks.

The set of descriptors (computed for each file) were attributed directly to the networks' input and the stopping criteria for training, used in our experiments, was the minimum error (1\%) and the maximum number of iterations allowed (100.000 epochs).

${ }^{3}$ The term normalization refers to the operation of correcting the amplitude of EEG recordings in which the maximum amplitude is greater than the one of a paroxysm $( \pm 200 \mu \mathrm{V})$. The applied correction is the ratio between the signal and its mean value. 
The best results obtained after the simulations with all these networks are presented in Table 3, where the following statistical indices can be observed:

- $\quad$ Success rate (SR);

- $\quad$ True positive $(\mathrm{TP})$, true negative (TN), false positive (FP) and false negative (FN);

- $\quad$ Sensibility (SE) e specificity (SP);

- $\quad$ Positive predictive value (PPV) and negative predictive value (NPV).

\begin{tabular}{|c|c|c|c|c|c|c|c|c|c|}
\hline ANN specifications & SR & $\mathrm{TP}$ & $\mathrm{TN}$ & FP & FN & SE & SP & PPV & NPV \\
\hline $8 \mathrm{~N}$ hidden $/ 10^{5}$ epochs ${ }^{a}$ & $81 \%$ & 27 & 19 & 4 & 3 & 0,90 & 0,83 & 0,87 & 0,86 \\
\hline 9N hidden / $10^{5}$ epochs ${ }^{a}$ & $79 \%$ & 27 & 20 & 3 & 3 & 0,90 & 0,87 & 0,90 & 0,87 \\
\hline $8 \mathrm{~N}$ hidden / $10^{5}$ epochs ${ }^{\mathrm{a}, \mathrm{c}}$ & $79 \%$ & 27 & 16 & 7 & 3 & 0,90 & 0,70 & 0,79 & 0,84 \\
\hline $8 \mathrm{~N}$ hidden $/ 10^{5}$ epochs ${ }^{b}$ & $80 \%$ & 18 & 24 & 1 & 2 & 0,90 & 0,96 & 0,95 & 0,92 \\
\hline 9N hidden / 11863 epochs $b$ & $89 \%$ & 17 & 24 & 1 & 3 & 0,85 & 0,96 & 0,94 & 0,89 \\
\hline 9N hidden / 12026 epochsb,c & $68 \%$ & 19 & 19 & 6 & 1 & 0,95 & 0,76 & 0,76 & 0,95 \\
\hline
\end{tabular}

a Training and test with files from composition I.

$\mathrm{b}$ Training and test with files from composition II.

c The input stimuli was normalized.

Table 3. Best results achieved with the Artificial Neural Networks created

According to results presented in Table 3 the use of files with signals classified by the occurrence of paroxysms showed success rate (the correct identification of test signals) of $79 \%$ whereas with the files of the composition II this rate was around $90 \%$. The best network implementations for each type of files showed sensitivity of $90 \%$ and $85 \%$ and specificity of $87 \%$ and $96 \%$.

The effect of normalizing the network's input stimuli that we observed during the simulations was a reduction in the specificity values due to the number of false positives generated (for example, for the network with nine hidden neurons the false positives increased from one to six).

\section{Conclusions}

The use and determination of morphological descriptors seems to be simple because it is a direct data collection with relatively basic calculations such as, for example, calculating the dimensions of amplitude and duration of the event. However, this process requires a priori knowledge of information about the system or entity which characteristics will be cataloged. In other words, for the case of automatic detection of epileptiform events in EEG recordings is necessary to carry out preliminary studies about the morphology of the signals to be analyzed. Another significant aspect when using morphological descriptors is the assessment of the selected descriptors as input of the classifier used. It is important to perform an evaluation to demonstrate the contribution of each descriptor for the capability of the ensemble in making the distinction between events of interest. In this study we used correlation analysis and Hotelling's $\mathrm{T}^{2}$ test to identify which descriptors could be excluded from the created set in order to provide a performance improvement of the automatic detection process. The methods applied for this assessment did not result in significantly high improvements in the automatic detection, but this does not invalidate its use because the classifier (neural network) used on the experiments showed promising results. 
Thus, it becomes necessary to study other advanced and robust analysis tools that can within a tolerance (error) threshold, provide more consistent results. Therefore we are using multivariate analysis (Principal Component Analysis, Independent Component Analysis) alone or in combination with other statistical techniques for assessing the relevance of the descriptors in attempt to optimize the size of the set needed to perform automatic detection through neural networks (or other classifier) without causing significant performance loss for the system in which the descriptors are inserted.

\section{References}

Abibullaev B.; Seo H. D. \& Kang W. (2009a) A Wavelet Based Method for Detecting and Localizing Epileptic Neural Spikes in EEG. Proceedings of the 2nd International Conference on Interaction Sciences: Information Technology, Culture and Human. pp. 702-707, 9781605587103, Seoul, Korea, November 2009, ACM, New York.

Abibullaev B.; Kim M. S. \& Seo H. D. (2009b) Seizure Detection in Temporal Lobe Epileptic EEGs Using the Best Basis Wavelet Functions. Jornal of Medical Systems. Vol.34, No.4, May 2009, pp. 755-765, 0148-5598.

Adeli, H.; Zhou, Z. \& Dadmehr, N. (2003). Analysis of EEG records in an epileptic patient using wavelet transform. Jornal of Neuroscience Methods. Vol.123, February 2003, pp. 69-87, 0165-0270.

Argoud, F.I.M.; De Azevedo, F.M.; Marino Neto, J. \& Grillo, E. (2006). SADE3: an effective system for automated detection of epileptiform events in long-term EEG based on context information. Medical \& Biological Engineering \& Computing. Vol.44, No.6, June 2006, Springer, pp. 459-470, 0140-0118.

Boos, C. F.; Pereira, M. C. V.; Argoud, F. I. M.; Azevedo, F. M.. (2010a). Analysis and definition of morphological descriptors for automatic detection of epileptiform events in EEG signals with artificial neural networks. Proceedings of the 3rd IEEE International Conference on Computer Science and Information Technology. Vol.5, pp. 349-353, 978-1-4244-5537-9, Chengdu, Sichuan, China, July 2010, IEEE Press.

Boos, C. F.; Pereira, M. C. V.; Argoud, F. I. M.; Azevedo, F. M.. (2010b). Morphological descriptors for automatic detection of epileptiform events. Proceedings of the 32nd Annual International Conference of the IEEE Engineering in Medicine and Biology Society. pp. 2435-2438, 978-1-4244-4124-2, Buenos Aires, Argentina, AugustSeptember 2010, IEEE Press.

Coimbra, A. J. F.; Marino J.; Freitas, C. G.; Azevedo, F. M., Barreto, J. M. (1994). Automatic Detection of Sleep-Waking States Using Kohonen Neural Networks. Proceedings of the I Congresso Brasileiro de Redes Neurais, Itajubá, Brazil, October 1994, pp. 327-331.

Diambra, L.; Fiqueiredo, J.C.B.; Malta,C.P. (1999). Epileptic Activity Recognition in EEG Recording, Physica A, Vol.273, No.3, November 1999, Elsevier, pp.495-505, 03784371.

Eberhart, R \& Dobbins, R. (1990). Neural Network PC Tools: A Practical Guide. Academic Press, 0122286405, San Diego, California.

Esteller, R. (2000). Detection of Seizure Onset in Epileptic Patients from Intracranial EEG Signal. PhD. Thesis, School of Electrical and Computer Engineering Georgia Institute of Technology, 2000.

Fisher, R.S.; Boas, W.E.; Blume,W., Elger, C.; Genton, P.; Lee, P.; Engel Jr, J. (2005) Epileptic Siezures and Epilepsy: Definitions Proposed by the International League Against 
Epilepsy (ILAE) and International Bureau for Epilepsy (IBE). Epilepsia, Vol.46, No.4, March 2005, p.470-472, 1528-1167.

Gotman, J. (1982). Automatic recognition of epileptic seizures in the EEG, Electroencephalography and Clinical Neurophysiology, Vol.54, No.5, November 1982, pp. 530-540, 0013-4694.

Gotman, J.; Gloor, P. (1976). Automatic recognition and quantification of interictal epileptic activity in the human scalp EEG, Electroencephalography and Clinical Neurophysiology, V.41, No.5, November 1976, pp. 513-529, 0013-4694.

Guedes, J. R. ; Pereira, M. C. ; Azevedo, F. M. ou de Azevedo, F.M. (2002). Parameterization of the EEG Signal Applied to the Detection of Epileptiform Events. Proceedings of the 2nd European Medical and Biological Engineering Conference, Vol.3(1), pp. 444-445, Vienna, Austria, December 2002, IFMBE.

Halford, J. J. (2009). Computerized epileptiform transient detection in the scalp electroencephalogram: Obstacles to progress and the example of computerized ECG interpretation. Clinical Neurophysiology, Vol.120, No.11, November 2009, pp. 1909-1915, 1388-2457.

Haykin, S. (1994) Neural Networks: A Comprehensive Foundation, Macmilliam College Publishing Company, 0-13-226556-7, Englewood Cliffs.

Hoef, L.; Elgavish, R.; Knowlton, R. C. (2010). Effect of Detection Parameters on Automated Electroencephalography Spike Detection Sensitivity and False-Positive Rate. Journal of Clinical Neurophysiology, Vol.27, No.1, February 2010, pp. 12-16, 0736-0258

Hoffmann, K.; Feucht, M.; Witte, H.; Benninger, F \& Bolten, J. (1996). Analysis and classification of interictal spikes discharges in Benign Partial Epilepsy of Childhood on the basis of the Hilbert transform, Neuroscience Letters, Vol. 211, No.3, June 1996, pp. 195-198, 0304-3940.

Indiradevi, K.P.; Elias, E.; Sathidevi, P.S.; Nayak, S.D. \& Radhakrishnan, K. (2008). A multilevel wavelet approach for automatic detection of epileptic spikes in the electroencephalogram, Computers in Biology and Medicine, V.38, No.7, July 2008, pp. 805-816, 0010-4825.

Kalayci, T. \& Özdamar, O. (1995). Wavelet preprocessing for automated neural network detection of EEG spikes, IEEE Engineering in Medicine and Biology Magazine, Vol.14, No.2, March 1995, pp. 160-166, 0739-5175.

Kaneko, H.; Suzuki, S. S.; Akamatsu, M. (1999). Multineuronal Spike Classification Based on Multisite Electrode Recording, Whole-Waveform Analysis, and Hierarchical Clustering. IEEE Transactions on Biomedical Engineering, Vol.46, No.3, March 1999, pp. 280-290, 0018-9294.

Khan, Y.U. \& Gotman, J. (2003). Wavelet based automatic seizure detection in intracerebral electroencephalogram, Clinical Neurophysiology, Vol.114, No.5, May 2003, pp. 898908, 1388-2457.

Kim, K. H.; Kim, S. J. (2000). Neural Spike Sorting Under Nearly 0-dB Signal-to-Noise Ratio Using Nonlinear Energy Operator and Artificial Neural-Network Classifier. IEEE Transactions on Biomedical Engineering, Vol.47, No.10, October 2000, pp. 1406-1411, 1406-1411.

Kumar, S. P.; Sriraam, N.; Benakop, P.G.; Jinaga, B.C. (2010). Entropies based detection of epileptic seizures with artificial neural network classifiers, Expert Systems with Applications, Vol.37, No.4, April 2010, pp. 3284-3291, 0957-4174. 
Litt, B.; Esteller, R; Echauz, J; D'Alessandro, M.; Shor, R.; Henry, T.; Pennell, P.; Epstein, C.; Bakay, R.; Dichter, M. \& Vachtsevanos, G. (2001). Epileptic Seizures May Begin Hours in Advance of Clinical Onset: A Report of Five Patients, Neuron, Vol.30, No.1, April 2001, pp. 51-64, 0896-6273.

Liu, H.S.; Zhang, T. \& Yang, F.S. (2002). A multistage, multimethod approach for automatic detection and classification of epileptiform EEG, IEEE Transactions on Biomedical Engineering, Vol. 49, No.12, December 2002, pp. 1557-1566, 0018-9294.

Malmivuo, J.; Plonsey, R. (1995). Bioelectromagnetism: Principles and Applications of Bioelectric and Biomagnetic Fields, Oxford University Press, 0-19-50-5823-2, New York.

Mohamed, N.; Rubin, D.M. \& Marwala, T. (2006). Detection of epileptiform activity in human EEG signals using Bayesian neural networks, Neural Information Processing Letters and Reviews, Vol.10, No.1, January 2006, pp. 231-237, 1738-2572.

Naghsh-Nilchi, A.R. \& Aghashahi, M. (2010). Epilepsy seizure detection using eigen-system spectral estimation and Multiple Layer Perceptron neural network, Biomedical Signal Processing and Control, Vol.5, No.2, April 2010, pp. 147-157, 1746-8094.

Niedermeyer, E. \& Silva F. L. (1993). Electroencephalography: Basic Principles, Clinical Applications, and Related Fields. Williams \& Wilkins, 0-683-06511-4, Philadelphia.

Ocak, H. (2008). Automatic detection of epileptic seizures in EEG using discrete wavelet transform and approximate entropy, Expert Systems with Applications, Vol.36, No.2, Part 1, March 2009, pp. 2027-2036, 0957-4174.

Ocak, H. (2008). Optimal classification of epileptic seizures in EEG using wavelet analysis and genetic algorithm, Signal Processing, Vol.88, No.7, July 2008, pp. 1858-1867, 0165-1684.

Oweiss, K.G. \& Anderson, D.J. (2001). Noise reduction in multichannel neural recordings using a new array wavelet denoising algorithm, Neurocomputing,Vol.38-40, June 2001, pp. 1687-1693, 0925-2312.

Pang, C.C.C.; Upton, A.R.M.; Shine, G. \& Kamath, M.V. (2003). A Comparison of Algorithms for Detection of Spikes in the Electroencephalogram, IEEE Transactions on Biomedical Engineering, Vol.50, No.4, April 2003. pp. 521-526, 0018-9294.

Pereira, M. C. V. (2003). Avaliação de técnicas de pré-processamento de sinais do EEG para detecção de eventos epileptogênicos utilizando redes neurais artificais. Thesis (PhD), Biomedical Engineering Institute, University Federal of Santa Catarina, 2003.

Pereira, M. C. V.; Azevedo, F.M. \& Argoud, F. I. M. (2003). Investigation About PreProcessing in the Input of an Artificial Neural Network for Analysis of Epileptogenic Events in EEG Signals. Proceedings of the World Congress on Medical Physics and Biomedical Engineering, pp.103-103, Sydney, Australia, IFMBE.

Pillay, J. \& Spearling, M. R. (2006). Interictal EEG and the Diagnosis of Epilepsy, Epilepsia, Vol.47, No.1, October 2006, pp. 14-22, 1528-1167.

Quiroga, R. Q. (1998). Quantitative Analysis of EEG Signals: Time-Frequency Methods and Chaos Theory. Thesis (PhD), Institute of Physiology, Medical University Lübeck and Institute of Signal Processing, Medical University Lübeck, 1998

Quiroga, R.Q.; Sakowitz, O.W.; Basar, E. \& Schürmann, M. (2001). Wavelet Transform in the analysis of the frequency composition of evoked potentials, Brain Research Protocols, Vol.8, No.1, August 2001, pp. 16-24, 1385-299X.

Saab. M. E. \& Gotman, J. (2005). A system to detect the onset of epileptic seizures in scalp EEG, Clinical Neurophysiology, Vol.116, No.2, February 2005, pp. 427-442, 1388-2457. 
Sanei, S. \& Chambers, J.A. (2007). EEG Signal Processing, John Wiley \& Sons, 978-0-47002581-9, West Sussex.

Scolaro, G.R. \& Azevedo, F.M. (2010). Classification of Epileptiform Events in Raw EEG Signals using Neural Classifier, Proceedings of the 3rd IEEE International Conference on Computer Science and Information Technology. Vol.5, pp. 368-372, 978-1-4244-5537-9, Chengdu, Sichuan, China, July 2010, IEEE Press.

Sovierzoski, M. A. (2009). Avaliação de descritores morfológicos na identificação de eventos epileptiformes. Thesis (PhD), Biomedical Engineering Institute, University Federal of Santa Catarina, 2009.

Srinivasan, V.; Eswaran, C. \& Sriraam, N. (2007). Approximate Entropy-Based Epileptic EEG Detection Using Artificial Neural Networks, IEEE Transactions on Information Technology in Biomedicine, Vol.11, No.3, May 2007, pp.288-295, 1089-7771.

Subasi, A. (2007). Application of adaptive neuro-fuzzy inference system for epileptic seizure detection using wavelet feature extraction, Computers in Biology and Medicine, Vol.37, No.2, February 2007, pp. 227-244, 0010-4825.

Tzallas, A.T.; Karvelis, P. S.; Katsis, C. D.; Fotiadis, D.I., Giannopoulus, S. \& Konitsiotis, S. (2006). A Method for Classification of Transient Events in EEG Recordings: Application to Epilepsy Diagnosis, Methods of Information in Medicine, Vol.45, No.6, March 2006, pp. 610-621, 0026-1270.

Übeyli, E. D. (2009). Statistics over features: EEG signals analysis, Computers in Biology and Medicine, Vol.39, No.8, August 2009, pp. 733-741, 0010-4825.

Walczak, S. \& Nowack, W. J. (2001) An Artificial Neural Network Approach to Diagnosing Epilepsy Using Lateralized Bursts of Theta EEGs, Journal of Medical Systems, Vol.25, No.1, February 2001, pp. 1-22, 0148-5598.

Webber, W. R. S.; Litt, B.; Wilson, K. \& Lesser, R. P. (1994). Practical Detection of Epileptiform Discharges (EDs) in the EEG Using an Artificial Neural Network: a Comparison of Raw and Parameterized EEG Data, Electroencephalography and Clinical Neurophysiology, Vol.91, No.3, September 1994, pp. 194-204, 0013-4694.

Wilson, S. B.; Emerson, R. (2002). Spike Detection: a Review and Comparison of Algorithms, Clinical Neurophysiology, Vol.113, No.12, December 2002, pp. 1873-1881, 1388-2457.

Wilson, S.B.; Scheuerb, M.L.; Emerson R.G. \& Gabor, A.J. (2004). Seizure detection: evaluation of the Reveal algorithm, Clinical Neurophysiology, Vol.115, No.10, October 2004, pp. 2280-2291, 1388-2457.

Zurada, J. M. (1992). Introduction to Artificial Neural Systems, West Publishing Company, 0314-93391-3, St. Paul. 


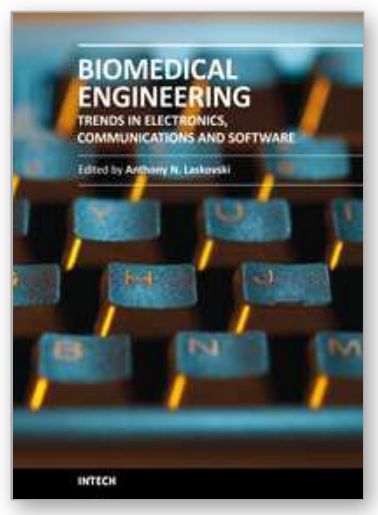

\author{
Biomedical Engineering, Trends in Electronics, Communications \\ and Software \\ Edited by Mr Anthony Laskovski
}

ISBN 978-953-307-475-7

Hard cover, 736 pages

Publisher InTech

Published online 08, January, 2011

Published in print edition January, 2011

Rapid technological developments in the last century have brought the field of biomedical engineering into a totally new realm. Breakthroughs in materials science, imaging, electronics and, more recently, the information age have improved our understanding of the human body. As a result, the field of biomedical engineering is thriving, with innovations that aim to improve the quality and reduce the cost of medical care. This book is the first in a series of three that will present recent trends in biomedical engineering, with a particular focus on applications in electronics and communications. More specifically: wireless monitoring, sensors, medical imaging and the management of medical information are covered, among other subjects.

\title{
How to reference
}

In order to correctly reference this scholarly work, feel free to copy and paste the following:

Christine F. Boos, Fernando M. de Azevedo Geovani R. Scolaro and Maria do Carmo V. Pereira (2011). Automatic Detection of Paroxysms in EEG Signals Using Morphological Descriptors and Artificial Neural Networks, Biomedical Engineering, Trends in Electronics, Communications and Software, Mr Anthony Laskovski (Ed.), ISBN: 978-953-307-475-7, InTech, Available from:

http://www.intechopen.com/books/biomedical-engineering-trends-in-electronics-communications-andsoftware/automatic-detection-of-paroxysms-in-eeg-signals-using-morphological-descriptors-and-artificial-neura

\section{INTECH}

open science | open minds

\author{
InTech Europe \\ University Campus STeP Ri \\ Slavka Krautzeka 83/A \\ 51000 Rijeka, Croatia \\ Phone: +385 (51) 770447 \\ Fax: +385 (51) 686166 \\ www.intechopen.com
}

\author{
InTech China \\ Unit 405, Office Block, Hotel Equatorial Shanghai \\ No.65, Yan An Road (West), Shanghai, 200040, China \\ 中国上海市延安西路65号上海国际贵都大饭店办公楼405单元 \\ Phone: +86-21-62489820 \\ Fax: +86-21-62489821
}


(C) 2011 The Author(s). Licensee IntechOpen. This chapter is distributed under the terms of the Creative Commons Attribution-NonCommercialShareAlike-3.0 License, which permits use, distribution and reproduction for non-commercial purposes, provided the original is properly cited and derivative works building on this content are distributed under the same license. 